Recherches en sciences sociales sur Internet

$8 \mid 2019$

Genre \& Internet. Sous les imaginaires, les usages ordinaires

\title{
Se réunir pour jouer
}

Les LAN parties entre ajustements et réaffirmation des identités genrées Playing with gender : LAN party between adjustments and reassertion of gender identities

Johann Chaulet et Jessica Soler-Benonie

\section{(2) OpenEdition}

\section{Journals}

Édition électronique

URL : http://journals.openedition.org/reset/1309

DOI : $10.4000 /$ reset.1309

ISSN : 2264-6221

\section{Éditeur}

Association Recherches en sciences sociales sur Internet

Référence électronique

Johann Chaulet et Jessica Soler-Benonie, «Se réunir pour jouer », RESET [En ligne], 8 | 2019, mis en ligne le 01 juin 2019, consulté le 05 juin 2019. URL : http://journals.openedition.org/reset/1309 ; DOI $10.4000 /$ reset.1309

Ce document a été généré automatiquement le 5 juin 2019.

(c) Association Recherches en sciences sociales sur Internet 


\section{Se réunir pour jouer}

Les LAN parties entre ajustements et réaffirmation des identités genrées

Playing with gender: LAN party between adjustments and reassertion of gender identities

Johann Chaulet et Jessica Soler-Benonie

\section{Introduction}

1 En France, comme dans d'autres pays, le jeu vidéo constitue une pratique éminemment genrée qui, traditionnellement, se trouve associée à l'univers masculin. Les hommes sont, par exemple, plus nombreux que les femmes à déclarer y jouer (Donnat, 2008) et sont très majoritaires dans les métiers de conception (voir chiffres baromètre annuel du jeu vidéo en France, SNJV 2017) ${ }^{1}$. Pourtant, la littérature française consacrée spécifiquement au rapport qu'entretiennent le genre et les jeux vidéo reste encore peu développée. L'androcentrisme n'a pas épargné ce domaine de recherche où les femmes sont souvent invisibles et où le genre est fréquemment relégué au rang de variable d'analyse. L'ouvrage dirigé par Fanny Lignon (2015) fait exception et pointe de multiples pistes de recherche. Si la nécessité d'étudier les imaginaires genrés véhiculés par les jeux a été largement démontrée (Triclot, 2015) peu d'enquêtes en France portent, en revanche, sur la production du genre en situation de pratique des jeux vidéo. C'est précisément l'objet de cet article qui s'intéressent aux rapports de genre « en train de se faire » en terrain geek. L'objectif est d'analyser comment ils se construisent par le biais d'une reproduction stéréotypée des rôles de chacun'e. En effet, si les femmes sont présentes sur notre terrain, elles participent à affirmer un certain virilisme. La figure du geek et ses nombreuses représentations et stigmates (addition, isolement, éternel célibat) ne semblent pas sans conséquences, nous le verrons, sur la façon dont les rapports de genre sont mobilisés dans le cours de l'action. Ils participent selon de nombreux mécanismes à sa revalorisation de cette figure. Aller vers une grande mixité ne semble donc pas remettre en cause mais plutôt renforcer le caractère profondément ancrée de la domination masculine dans l'univers des jeux vidéo et ses pratiques. 
2 Cet article entend pointer un certain nombre de ses enjeux en empruntant aux travaux qui ont analysés les rapports de genre dans des espaces dominés par les hommes. Ces derniers sont variés et leurs analyses s'inscrivent dans des champs qui le sont tout autant, allant de la sociologie des professions (Zolesio, 2009, 2012) ou encore celle du sport (Saouter, 2010). Ces enquêtes mobilisent de nombreuses données en situation montrant comment les femmes investissent et s'intègrent dans des espaces de pratique masculin en occupant certains types de posture (spectatrices, gestionnaire du linge, Saouter, ibid.) ou encore en s'adaptant aux codes virils en présence (humour grivois, valeurs viriles, Zolesio, ibid.). D'autres travaux sont plus directement en lien avec le genre et l'usage des technologies informatiques (Auray, 2002, 2007 ; Collet, 2006 ; Gourarier, 2014, 2017) ; ont, eux aussi, naturellement nourri notre analyse. L'ensemble de ces références pointent les liens structurant entre masculinité et culture geek allant des espaces de démos (Auray, ibid.), de drague (Gourarier, ibid.) aux écoles d'informatiques (Collet, ibid.). Si ces références détaillent les mécanismes de l'entre-soi masculin, notre analyse montre comment il se donne à voir et s'adapte au sein de pratiques plus quotidiennes et présentant davantage mixité.

Pour cette analyse, nous mobilisons une enquête de terrain fondée principalement sur l'observation in situ de ce qu'on appelle des LAN parties (pour Local Arena Network). Des LAN parties sont des regroupements de joueureuse's qui, durant un ou deux jours généralement, se réunissent, équipée's de leurs ordinateurs personnels sur un même réseau local, pour prendre part à un tournoi de jeu vidéo. Ils et elles s'affrontent dans le cadre d'un jeu auquel ils et elles ont par ailleurs, pour la plupart, l'habitude de jouer en ligne à domicile. Si on considère que le genre est, au moins dans une certaine mesure, un «accomplissement local» sans cesse rejoué, reconstruit et réaffirmé (West \& Zimmerman, 1987), alors les LAN parties peuvent être pensées comme le théâtre - et donc un lieu d'observation particulièrement pertinent - de représentations du genre qui régulent les rôles que doivent jouer les hommes et les femmes, et que ce théâtre contribue activement à construire. Les LAN parties apparaissent en effet comme des lieux de mises en scène particulièrement prégnants des rapports sociaux de genre qu'elles contribuent en retour à faire exister. L'observation de ces évènements collectifs permet de mieux comprendre certains des ressorts interactionnels d'une pratique ordinaire, le jeu vidéo, habituellement enfermée au sein du domicile mais qui dessine pourtant d'importants enjeux relationnels, en termes de genre notamment. Elle permet en effet de rendre visible des rapports de genre que la distance des corps pourrait, au moins en partie, rendre difficilement accessibles voire invisibles.

$4 \quad$ Pour étudier cette production du genre lors des LAN parties, trois méthodes ont été mises en œuvre dont principalement l'observation. En France, peu de travaux sur les jeux vidéo mobilisent, en tant que méthodologie principale, l'observation physique in situ de pratiques en «train de se faire» privilégiant des approches quantitatives et/ou qualitatives par entretiens et avec une immersion quotidienne online (Berry, 2011; Boutet, 2012 ; Coavoux \& Gerber, 2016; Zabban, 2011). Ces enquêtes s'accordent pour décrire des mondes virtuels complexes (Zabban, 2009) supports et créateurs de sociabilités. Toutefois, ces mondes virtuels ne sont pas les seuls à prendre en compte. En effet, d'autres espaces, à l'image des LAN parties, participent au quotidien de pratique des joueur-euse's. Ces évènements débordent le cadre du jeu et ses représentations saisies habituellement par le biais d'ethnographies en ligne et d'entretiens. Les tournois 
amateurs offrent eux la possibilité d'observer à la fois les sociabilités mais également la pratique collective et ordinaire en train de se faire.

5 Les observations ici réalisées concernent des tournois du jeu League of Legends ${ }^{2}$. Il s'agit d'un jeu en ligne, gratuit et multijoueureuse's où s'affrontent des équipes, composées de cinq personnes, lors de parties qui durent en moyenne 20 minutes. Le but principal du jeu League of Legends est d'envahir le territoire ennemi en détruisant le cœur de sa base nommée le Nexus. Il propose d'incarner plus d'une centaine de personnages nommés « champions " aux caractéristiques variées occupant des rôles de défensifs et offensifs. League of Legends est un jeu d'affrontement en arène (MOBA) ${ }^{3}$ où se battre avec les adversaires constitue le principal mécanisme du gameplay. Faire reculer, affaiblir et tuer les ennemi-e's présent-e's occupent l'essentiel du temps de jeu. La victoire sur le camp adverse est facilitée par une bonne maitrise de sa position dans l'équipe autant que celle de son/sa championne et de ses spécificités. Les tournois observés ont eu lieu dans deux amphithéâtres de l'université toulousaine de sciences, réaménagés pour l'occasion. Plus précisément, cinq séances d'observations ont été réalisées par les deux auteur·e· ${ }^{4}$. Elles ont eu lieu dans le cadre de compétitions organisées par l'association étudiante Comput Yourself à Toulouse. Une première, exploratoire, a été mise en place via le collectif de chercheure ComUniTIC ${ }^{5}$ et en partenariat avec une école d'informatique locale dans le but de tester la grille d'observation. Une attention particulière a été portée autant aux temporalités de l'évènement (installation, phase de jeu et moment de pauses et d'attente) qu'à l'ensemble des interactions visibles pendant les parties mais aussi et surtout « hors » et "autour» du jeu. La principale volonté était de suivre les LAN parties le plus fidèlement possible en limitant toutes formes d'interférence des actions et interactions en cours. Les accords de l'association et de l'école informatique ont été obtenus en amont afin de mettre en place les séances d'observation. Le statut de l'enquêteur et de l'enquêtrice a été relevé lors des rares demandes directes de joueureuse's présentant une curiosité au sujet de notre présence. Généralement, ces dernière's se sont alors montrée-s peu étonnée's de nous voir dans cet espace.

6 À ce matériau s'ajoute des entretiens informels avec les participante's et les organisateurs lors desquels les enquêteur.trice's ont pu confronter leurs interrogations spontanées aux enquêtée's. Un court questionnaire a également été réalisé et distribué aux joueureuse's participante's au tournois ${ }^{6}$. L'objectif de ce dernier était de caractériser la population présente (âge et situation professionnelle, genre, lieu de résidence) et les modalités de participation (si la personne est venue seule ou en groupe ; la motivation de sa participation etc.) tout en donnant des indices sur le coût du matériel personnel des joueureuse' ${ }^{7}$.

7 Le questionnaire montre que la population observée est à l'image des lieux d'observation, à savoir une université de sciences « dures » et techniques. Les 195 répondant·ess sont très largement jeunes ( $45 \%$ âgée's de 18 à 20 ans ${ }^{8}$ ) et étudiante-s ( $\left.85 \%\right)$. La similitude avec les occupants habituels de ces amphithéâtres de chimie et d'ingénierie ne s'arrête pas là puisque 96 \% des participants sont des hommes. De même, une écrasante majorité des participante's $(91 \%)$ connaissent physiquement au moins un membre de leur équipe avant leur arrivée dans la compétition. Invitée's à hiérarchiser les motivations de leur participation entre des aspirations sociales ou compétitives, les joueur-euse's déclarent très majoritairement se rendre à ces événements " passer un moment entre ami-e·s » (53 $\%$ du choix numéro 1 et $28 \%$ du second) ainsi que pour «s'amuser » $(27 \%$ du choix numéro 1 et $44 \%$ du second). 
Si les motivations sociales semblent donc très majoritaires, la dimension compétitive ne disparaît pas puisque, une fois passées les aspirations relationnelles, $40 \%$ déclarent souhaiter " réaliser une bonne performance ». Le travail de perfectionnement qu'appelle une pratique intensive et compétitive du jeu se retrouve dans des formes de jeux plus traditionnelles comme la pratique du jeu d'échec. Deux enquêtes (Fine, 2015 ; Wendling, 2002), se rapprochent de notre démarche immersive et de certains de nos résultats. Elles mettent en avant l'importance de l'engagement et de la performance (réflexion, concentration, entrainement, rapport au corps) dans la pratique du jeu tout en soulignant que ces espaces compétitifs donnent à voir des aspects profondément culturels et sociaux (Fine, 2015; Wendling, 2002). Les compétitions vidéoludiques (T.L. Taylor, 2012) et plus récemment celle autour de League of Legends ont elles aussi fait l'objet de travaux autour du monde du sport électronique (Besombes, 2016 ; N. Taylor, 2015) qui mettent en avant ces deux aspects: expertise des joueureuse's et sociabilités centrales au sein de la pratique vidéoludique.

9 Nous allons voir ici combien cet entre-soi est un entre-soi masculin, empli des valeurs et représentations d'un certain type de masculinité faisant ici figure de référence à l'aune de laquelle chacune doit se positionner. Après avoir décrit les façons « d'être des hommes » en LAN parties, nous examinerons les positions complexes voire contradictoires qu'occupent les femmes en analysant l'éventail limité des rôles qu'il leur est possible de jouer au sein de ces événements. Des formes et formats d'évaluation, de gratification et de rapprochement aux façons de se parler, de se placer et de se mouvoir, ce sont tout un ensemble de modes d'agir éminemment genrés qui sont mis en œuvre lors des parties mais aussi et surtout autour du jeu lui-même.

\section{Un univers largement masculin}

10 Dès leur arrivée, les personnes présentes et participant au tournois s'affairent afin de s'installer confortablement. L'importance accordée au matériel informatique est visible dans le soin apporté à l'ensemble de l'équipement (casques, tour, écrans, souris et tapis). Valises à roulettes, cartons d'origines et parfois même couvertures sont là pour garantir le transport dans de bonnes conditions d'un matériel important et de valeur. Certaines marques sont plébiscitées ; les conversations et commentaires vont bon train et portent souvent sur le matériel qu'on est en train de déballer. De nombreux joueurs (uniquement des hommes) arborent des T-shirts à l'effigie de tel ou tel fabricant de matériel participant ainsi, à leur façon, à confirmer l'importance du matériel nécessaire à la pratique compétitive en ligne et objet récurrent de préoccupation.

\section{Les LAN comme arène de démonstration d'habiletés techniques}

11 Une fois ce matériel installé et après la gestion souvent longue des incontournables problèmes de serveur, la compétition peut commencer. Chaque équipe désigne alors un capitaine $^{9}$; il sera chargé de communiquer avec le capitaine adverse et de de transmettre les résultats en fin de partie. Quand bien même il est difficile d'établir une corrélation irréfutable entre le genre et la fonction (en effet, la très faible participation numérique des joueuses $-4 \%$ de l'effectif total - est tout autant à prendre en considération) nous pouvons cependant noter que, durant l'ensemble de nos observations, seuls des hommes ont été désignés, au sein de leur équipe, comme leur représentant légitime. 
12 En jeu, la concentration se lit sur les visages, les regards sont multi-situés, repérant et anticipant l'ensemble des actions sur les écrans de jeu. Les mouvements sont exécutés avec rapidité et dextérité, fruits de la gestion de nombreux paramètres en simultané : gestion du personnage, de son or et de ses objets, action personnelle et coordination avec le reste de l'équipe sur la carte de jeu, vocalement ou par écrit. La gestion de cet multitude de paramètres et ce travail de coordination cognitive rappellent ceux des joueurs professionnel de jeux FPS ${ }^{10}$ décrit par T.L. Taylor (2012) au travers de la notion de « complexe circuit d'action».

Si les affrontements entre équipes au sein du tournoi constituent l'occasion principale pour faire état de ses capacités et compétences, les temps hors tournois sont eux aussi mobilisés par les joueureuse's pour faire démonstration - mais aussi, certainement, pour accroître et entretenir - leurs habiletés techniques. Il n'est pas rare de voir des jeux dont le principe même repose directement sur les réflexes, la rapidité et la précision du joueureuse. Osu !11 ou TrackMania ${ }^{12}$ deviennent alors de véritables " arènes » publiques des " habiletés techniques" des participants (Dodier, 1993). A la manière de la compétition décrite par Marie-Christine Legout dans les salles en réseaux, il s'agit de faire démonstration de ses aptitudes vidéoludiques devant une audience : ces joueurs " attendent que l'on reconnaisse leurs talents» (Legout, 2003: 142). L'importance du collectif et des audiences prend toute sa dimension avec la présence d'un joueur « semi professionnel " de renommée nationale lors de la quatrième LAN et rappelle l'importance, même au cours de rencontres physiques, de la réputation en ligne (T.L. Taylor, 2012 ; N. Taylor, 2015). De petits groupes de joueurs s'agrègent autour de lui qui viennent assister à ses prouesses. Ces arènes de démonstration des habiletés vidéoludiques, à la manière des compétitions sportives décrites par Pascal Duret (2009), mêlent concurrence, rivalité et entraide. Les interactions reposent autant sur la matérialité que sur le contact direct entre les corps des personnes en présence. Les joueureuse's ainsi réunie's reconnaissent mutuellement leurs valeurs et établissent ensemble une complicité reposant sur les prouesses accomplies par les un'e's et publiquement reconnues par les autres. Les valeurs et qualités mobilisées et mises en scène (compétition, dépassement de soi, combativité) sont des valeurs traditionnellement attachées au masculin (Baudelot \& Establet, 2007; Welzer-Lang, 1995) et font écho à celles acquises par les hommes lors de la socialisation différenciée et entrânant une bisegmentation des modèles sexués (Lahire, 2001). Nos résultats illustrent une certaine forme de masculinité par les pratiques (Connell, 2014), ici celle du jeu, qui se donne publiquement à voir à une échelle individuelle et collective. Ces espaces apparaissent en effet comme le moyen pour les participants de réaffirmer un maniement expert masculin de la technique et des outils. Chacun est invité à se mesurer, à faire démonstration, et à évaluer sa valeur au sein du collectif des hommes dont son habileté technique doit pouvoir lui permettre de prouver qu'il mérite d'occuper une place légitime.

Ces quelques observations ne sont pas sans rappeler, bien sûr, l'ensemble des travaux sur l'appropriation masculine des techniques et leurs usages. Des observations de l'anthropologue Paola Tabet (1998) sur les sociétés de chasseurs/cueilleurs décrivant la façon dont le maniement des outils les plus techniques et spécialisés sont réservés aux hommes aux travaux de Delphine Gardey (2005) sur le processus de sexualisation de la machine à écrire, les analyses s'accordent sur la dimension sociale et genrée des technologies, leurs pratiques et leurs usages. Concernant l'informatique et la pratique des jeux vidéo, des mécanismes identiques ont été décrits avec les figures archétypales du « 
passionné de jeux vidéo » (Peyron, 2011) ou du « virtuose d'informatique » (Auray, 2002) et des logiques virilistes qui se trouvent médiatisées par ces supports (Collet, 2006, T.L. Taylor, 2012). Cette figure d'excellence dans le domaine informatique associée au masculin porte néanmoins en elle son pendant négatif incarné par «l'adolescent accro » (Berry, 2012), représentation dévalorisante associant informatif et désocialisation et cristallisée autour de la figure du jeune passionné de technologies, isolé et connaissant des difficultés dans les rapports de séduction. Nous verrons comment ces deux facettes (excellence/désocialisation) irriguent directement les sociabilités ainsi que les rapports sociaux de genre visibles en LAN parties.

La démonstration d'une certaine virilité est donc visible au travers de parade de genre au sens goffmanien du terme comme « exposition publique de soi » (Goffman, 2004). Le corps peut sembler en parti délaissé dans l'élaboration de l'identité masculine, au contraire d'autres fiefs de virilité comme l'armée (Teboul, 2015) ou le rugby (Saouter, 2010). La parade se trouve en effet ici largement équipée par la technique et sa mise en scène qui médiatisent la performance. Pour autant, puisqu'il est directement impliqué et engagé dans le maniement des objets, le corps ne disparaît pas. Il existe au contraire et se trouve, lui aussi, mis en scène dans ce qui semble révéler la volonté d'un certain dépassement et de vouloir " tenir le coup " que les hommes joueurs et leurs différentes extensions rendent sans cesse visibles. Plus la compétition avance plus, les piles de canettes de Redbull sont érigées aux côtés des ordinateurs, symbole du marathon auquel les joueur'euse's s'apprêtent à prendre part. Ces constructions invariablement présentes dans toutes nos observations semblent témoigner d'une volonté de dépassement de soi, et font directement écho à la recherche de performance évoquée ici. Sur la photo ci-dessous, ce joueur semble se réclamer du groupe des "champions" ayant choisis, comme lui, la marque Asus. La pile de canettes de boissons énergisantes à portée de main affiche, tel un porte-parole, «I have no limit ». 
Illustration 1 : Un joueur en début de tournoi avec une pile de canettes de boissons énergisantes et une bouteille de rosé.

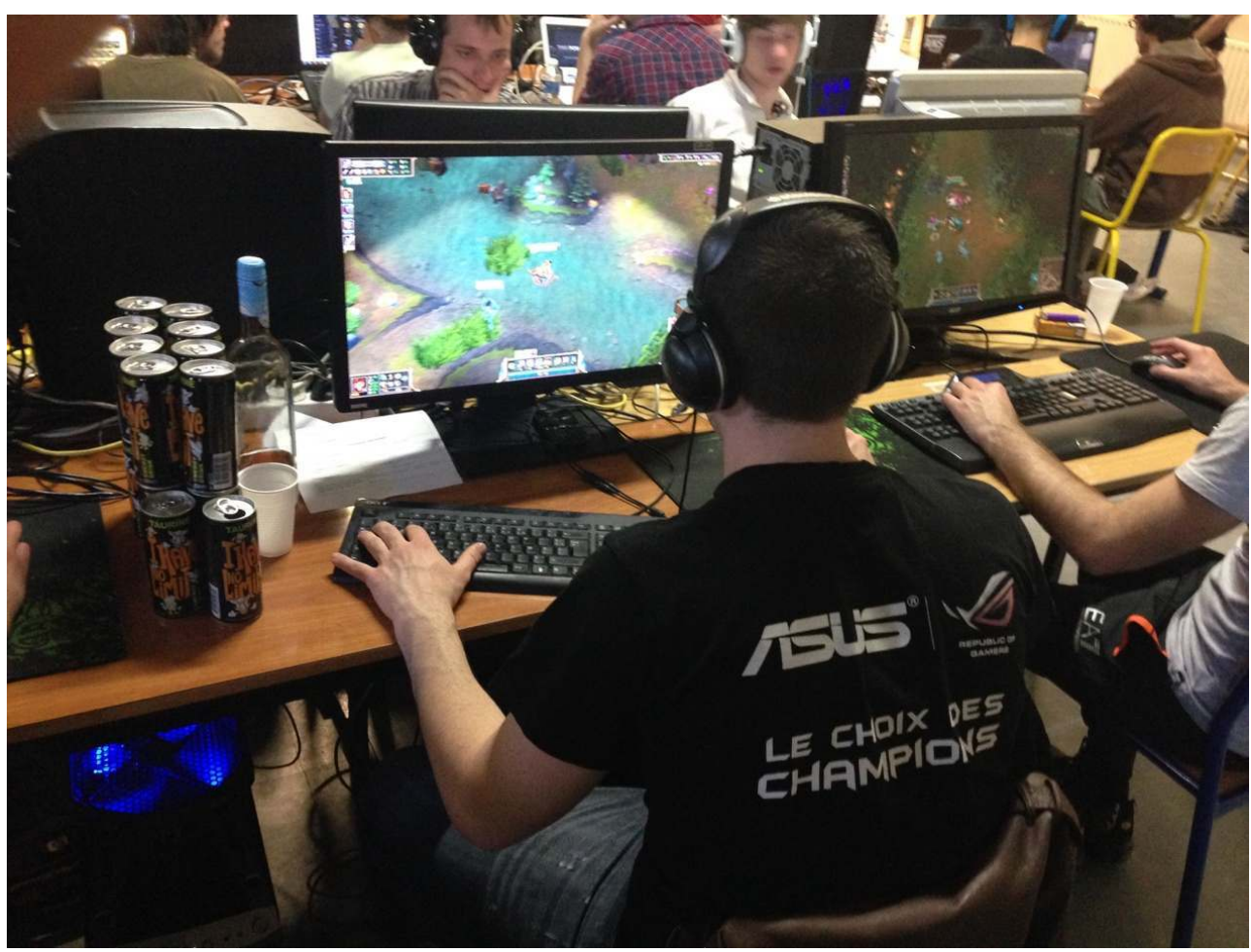

Source : photo réalisée par l'enquêteur LAN « CYL » 2014

16 À mesure que les heures passent, plutôt que d'être directement jetés, les emballages s'accumulent autour des postes de jeu et se font les témoins d'une certaine volonté de "tenir le rythme». Leur amoncellement sur les tables, généralement sous forme pyramidale, semble presque constituer une activité ludique à part entière. Si le dépassement physique de joueureuse's qui restent, des heures durant, assis devant leurs écrans, n'est pas directement visible, il se trouve ici visuellement et matériellement objectivé.

Boire de l'alcool (voir bouteille de rosé ci-dessus) et/ou fumer de la marijuana est également fréquent pendant le tournois. Loin d'être clandestine et en contradiction avec la recherche d'amélioration des performances, ces consommations collectives participent à la célébration en cas de victoire et sont toujours partagée sur le registre de la camaraderie entre hommes. En effet, bien que présentes lors de ces moments, les joueuses se cantonnent elles à la consommation de café et de boissons énergisantes.

Joueur 1 : bon on va rouler un buzz ou quoi?

Joueur 2 : plus tard quand on sera bien

Joueur 1 : je me fais chier là, je sais pas quoi faire

Joueur $2:$ m'en parle pas!

Joueuse : la prochaine fois on arrive à $11 \mathrm{~h} 30$.

(« La fille de l'équipe » intervient pour la 1ere fois) » (CYL, JC, Mars 2013).

« 15h30: Tu le sens le petit de la victoire? Un autre joueur lui répond: oui! Un autre rajoute : restez focus les mecs on a pas fini ». 5 min plus tard, tous se lèvent en sursaut, ils viennent de gagner le match. Salutations des adversaires. Ils se dirigent tous vers l'extérieur. Tous attendent le Joueur 4. Je réalise qu'il roule un joint. Il le partage avec ses partenaires d'équipe ». Je comprends au fur et à mesure 
que l'expression « c'est l'heure » signifie pour l'équipe le moment d'aller fumer de l'herbe » (CYL, JSB, Septembre 2015)

«Ils vont profiter d'une pause pour régler le problème de l'un d'entre eux ; un autre en profitera pour selon son expression : «aller chercher de la weed » (de la marijuana)» (CYL, JC, septembre, 2015)

« $18 \mathrm{~h} 15$ : Je sors fumer une cigarette. Nuage de fumée : ça sent l'herbe. Une équipe fume, le joint circule entre les joueurs. Une seule joueuse est au milieu : elle fume une cigarette son café à la main » (CYL, JSB, avril 2014).

\section{Vocabulaire distinctif} dans le registre de langue et le vocabulaire ainsi que dans « la gueule » et le volume sonore du discours (Teboul, ibid.) où être un gamer se montre aussi avec ostentation (Goffman, ibid.). Cette virilité donne un exemple du passage d'une « forme de masculinité offensive » (Bertrand et al, 2015) (agressivité, force physique) à une masculinité «maitrisée » où les hommes s'imposent par « le verbe " plus que par « le geste » (ibid.). Toutefois, « le geste » est, dans une certaine mesure, toujours présent et transposé au sein de cette " masculinité maîtrisée » où dimensions mentales et techniques s'entremêlent et s'incarnent dans la concentration et l'excellence du maniement des technologies.

Observer n'est pas seulement voir mais aussi entendre et (essayer de) comprendre. Tout comme l'expérience décrite par Vinciane Zabban concernant les MMOG, la découverte des LAN « pourrait à ce niveau être comparée à l'immersion dans un pays de langue et de culture totalement étrangère » (Zabban, 2007). De fait, écouter les joueurs parler entre eux peut s'avérer délicat pour l'observateur non averti tant sont nombreux les éléments de vocabulaire issus du jeu ou les abréviations propres à la communauté. Joueurs est ici sciemment employé au masculin. On peut noter, pour commencer, que les joueurs n'ont de cesse de s'interpeller en utilisant de manière quasi systématique le terme « mec ». Les joueuses quant à elles restent silencieuses, à plus forte raison quand il s'agit de faire quelque commentaire technique durant les parties.

« Trop de lag/j'ai 135 creeps/ essaye les shows run/ c'est pink wardé les bush/ y'a déjà

1-6 et un drake, je suis fier de ma game, GG » (LC et CYL, JC, mai et novembre 2013).

« Ils s'appellent "mec" à toutes les phrases » (CYL, JC, mars 2013).

Le vocabulaire spécifique que les joueurs mobilisent constamment, est composé, pour l'essentiel, de termes anglo-saxons directement tirés du jeu : «drake, creeps, pink wardé, bush » et du jargon informatique et vidéoludique : « lag, game, GG, config ». Un important effort de traduction est nécessaire pour le néophyte pour comprendre le contenu des conversations entre les joueureuse's, y compris en dehors des parties. En effet, le lexique mobilisé pendant les matchs contamine l'ensemble des échanges, dans l'enceinte de la compétition comme à l'extérieur. Outre sa complexité, qui instaure une barrière entre personne qui joue et non-joueureuse's - que seule l'expérience et le fait de faire partie intégrante de la communauté peut faire disparaître -, les joueur.euse's confèrent à l'usage de ce vocabulaire particulier un rôle fédérateur évident. Il constitue un moyen de faire groupe, les termes employés servant de points d'appui à des formes d'humour et de complicité totalement adossées à l'univers de League of Legends et à son vocabulaire. Les références au jeu sont nombreuses et quelques façons de faire ou styles de jeu archétypiques hissées au rang de références communes. Telle façon de jouer sera raillée, telle autre encensée. Qu'il s'agisse de célébrer une victoire ou de se plaindre d'une défaite, les réactions des joueurs sont vives et ces derniers s'assurent qu'ils seront largement vus 
et entendus. "Cette mise en scène excessive de soi », ce "débridement des émotions " comme relâchement du contrôle social constitue un trait caractéristique de ces compétitions (Auray 2007).

Il y a 5 min le joueur au pull rouge levait les bras en l'air et faisait semblant de crier.

Il vient de hurler « BOOOOM ! » et chantonne maintenant. Il se fait entendre. (CYL,

JC, Mars 2013)

21 Comme l'ont noté avant nous les auteurs ayant étudié la pratique des jeux vidéo en ligne (Auray, 2007 ; Berry, 2011) le registre de langue, lors des LAN également, est familier voire grossier. Dans ce type de rassemblements, « on ne se bat que dans l'arène virtuelle, on s'insulte copieusement mais dans une atmosphère amicale où tel est l'usage, ponctué par les rires »(Schmoll 2003: 47). Les joueurs se taquinent et se provoquent très régulièrement ; leurs réactions sont rendues visibles par des manifestations corporelles souvent fortes. Les cris sont fréquents lors des séquences de jeu particulièrement intenses. Si les insultes sont légion lors des parties, les façons de se parler sont au contraire très cordiales entre les matchs. Nous constatons en effet que les insultes restent, la plupart du temps, cartonnées aux moments de jeu, lorsque les parties sont en cours et que l'échange se fait au travers des écrans. Au contraire, les interactions entre les membres d'équipes opposées sont extrêmement cordiales et ritualisées ; le fair play est de rigueur et ceux qui se sont battus se félicitent mutuellement.

Plus la compétition avance plus le vocabulaire employé transforme la LAN en arène de démonstration virile. Un champ lexical sexuel est repérable, utilisé par tous, qui, associé au vocabulaire guerrier du jeu, afin de qualifier les adversaires ou décrire les actions en cours ou à entreprendre.

«J'vais le défoncer »; « viens, on va le niquer! »

«oh c'que tu lui as mis!» (5 fois entendu pendant la partie) (CYL, JC, novembre

2014)

Lors de manœuvres adverses désavantageuses, de mise en difficulté, les joueurs concurrents sont qualifiés, à voix haute, par des termes sexistes et homophobes :

« Ah la pute!»; « On s'est fait violer »; « Ah le PD!» (CYL, JSB, mars 2013)

«Allez, allez, allez! Encore 28 fight, on n'est pas des pédés!» (CYL, JC, novembre 2014)

"L'autre » fait office de figure repoussoir et il s'agit, ici, de femmes ou d'homosexuels (Clair, 2012). Comme le note Ducrocq, " le LAN semble répondre à un fort besoin de reconnaissance et d'intégration sociale chez les garçons à une période de leur existence où la construction identitaire passe par l'identification masculine à un groupe d'âge équivalent " (Ducrocq, 2011: 293). L'utilisation ordinaire de termes sexistes et homophobes est majoritaire et ne se voit opposé aucune forme de censure, il se retrouve également au sein d'autres univers masculins de compétition à l'image des jeux d'échecs (Fine, 2005; Wendling, 2002). Seul les hommes emploient ce type de termes et seul le volume sonore est éventuellement dénoncé auprès de l'organisation et peut faire l'objet de rappels à l'ordre.

\section{Entre-soi masculin}

Ce vocabulaire sexiste et sexuel opère donc ici un double rôle de rapprochement complice entre les hommes et de mise à distance de ceux qui n'en sont pas, les homosexuels ou les femmes. Cette fonction du vocabulaire et de l'humour à caractère sexuel a par ailleurs pu 
être repérée dans le cadre de milieux professionnels techniques traditionnellement masculins. Sylvie Cromer et Dominique Lemaire notent ainsi que « se distinguent deux grandes fonctions conférées à la sexualité, fonctions non exclusives, qui s'entremêlent et se relaient, l'une tournée vers la cohérence et la consistance du groupe masculin, l'autre visant à tenir à distance le groupe féminin intrusif. La sexualité, manifestée par des vantardises et des plaisanteries sexuelles ou encore l'usage de la pornographie, serait l'expression d'une marge de liberté conquise, un espace de desserrement des contraintes professionnelles, partie intégrante de stratégies de défense virilistes, individuelles ou collectives » (Cromer \& Lemaire, $2007: 69$ ).

Emmanuelle Zolesio effectue des constats similaires dans le milieu largement masculin de la chirurgie (Zolesio, 2009). Ici encore, l'une des fonctions de l'humour consiste justement à opérer des formes de reproduction et de sélection auprès de ceux qui veulent se réclamer du groupe des pairs. Ainsi note-t-elle que « les plaisanteries grivoises et sexistes contribuent efficacement à l'éviction des femmes " (: 124). De fait, comme dans nombre d'univers mettant en œuvre des habiletés techniques, les femmes sont assez largement exclues dans cet univers très masculin. Les dispositions dont il s'agit de faire usage et démonstration dans le cas des LAN parties s'apparentent assez largement à celle que requiert l'exercice de la profession de chirurgien, elle aussi éminemment masculine. En effet, tel que le décrit Emmanuelle Zolesio à la suite de Cassell, et Katz, « disposition à l'action, disposition au leadership autoritaire, dispositions combatives, disposition à l'endurance physique semblent nécessaires à l'exercice du métier chirurgical. Autant de dispositions socialement construites comme masculines et perçues comme telles.» (Zolesio, 2009 : 122).

Pourtant, nos observations nous permettent de constater que, même si minoritaires, les femmes sont bel et bien présentes dans ces espaces au sein desquels elles s'efforcent de trouver leur place en adaptant, bien souvent, leurs façons d'être et de faire aux normes et codes masculins en vigueur. En effet, "la marge de manœuvre féminine, face à la sexualité masculine, source de sociabilité masculine, mais potentiellement vecteur de violence et de handicap à l'intégration professionnelle, consiste à prendre appui sur cette représentation partagée (...) pour s'y orienter et trouver une place, sans péril pour son identité sexuée. Elle reste fort étroite » (Cromer \& Lemaire, 2007 : 70-71).

\section{Un répertoire limité de rôles pour les femmes}

Le langage participe à la mise en scène et en forme du groupe des joueurs pour lesquels l'enjeu consiste à faire la démonstration de leurs compétences. Il procède également de la mise en scène de leur virilité au sein d'un espace majoritairement masculin. Les LAN parties pourraient alors apparaitre comme un milieu parmi d'autres d'expression de la masculinité hégémonique (Bertrand, Court, Mennesson, Zabban, 2015) où s'entretiennent des rapports homosociaux « classiques ", à l'image d'un club de sport (Welzer-Lang, 1994). Cependant, même si elles restent largement minoritaires, les femmes participent à ces rassemblements d'e-sport (sport électronique) amateurs (Rabindra, Taylor, Hogan, 2015). Selon notre recensement les joueuses sont plus nombreuses en 2014 (8) qu'en 2012 (3). Toutefois, elles ne se retrouvent pas là uniquement en tant que participantes directes à la compétition. En effet, les femmes sont aussi présentes dans le corps organisationnel du collectif à l'origine de l'évènement et leur plus grand nombre compte parmi l'effectif du public venu assister à l'évènement. Historiquement, le changement de forme de ces 
espaces (plus ouverts, non commerçants) pourrait expliquer une arrivée plus importante de joueuses et de femmes spectatrices qu'elles ne l'étaient dans les espaces de jeu collectifs qu'étaient les cybercafés (Legout, 2003) où la présence de femmes était directement remarquée puis stigmatisée.

Outre le langage, l'examen plus détaillé des manières d'être et de jouer permet de saisir la façon dont l'ensemble des modes de participation des femmes en LAN parties sont le fruit de divisions sexuées des rôles. Nous allons voir comment ces dernières contribuent certainement à entretenir, voire à renforcer, des assignations de genre fortement palpables et visibles. Prendre part à une LAN parties signifie pour les femmes d'incarner une des trois figures que nous allons ici détailler et qui, aussi archétypiques soient-elles, résument assez fidèlement la gamme de rôle à disposition des femmes et qu'elles peuvent légitimement adopter lorsqu'elles prennent part à ces rassemblements.

\section{Filles du staff}

30 Si des hommes et des femmes composent le collectif d'organisateurs, les différents types de tâches à prendre en charge sont segmentés de manière relativement hermétique. Les quatre hommes sont responsables de la bonne marche de la technique et les femmes (deux à quatre selon les éditions) de l'accueil des joueurs puis de la vente, du ravitaillement et de la gestion du stock de snacks et boissons vendus sur place aux joueureuse's. L'enquêtrice s'étonnera auprès d'un des organisateurs - en feignant de pas avoir y prêter attention plus tôt - de l'absence de femmes au sein de l'équipe technique. Il lui sera répondu que « les filles du staff » (son expression) sont en couple avec des membres de l'association : elles sont là avant tout dans le but de " donner un coup de main ». On serait donc enclins à penser que, n'étant ni directement concernés par le tournoi ni membres de l'association, leur présence ne serait que ponctuelle. Les femmes du « staff » sont pourtant présentes, au même titre que les techniciens réseau, du samedi $8 \mathrm{~h} 30$ au dimanche $18 \mathrm{~h}$.

31 Après quelques heures écoulées et les premières phases de jeu entamées, les tensions dues à la mise en place et aux nombreux réglages techniques à opérer sur le réseau s'atténuent. Les bénévoles peuvent interagir plus sereinement entre eux/elles. Nous constatons en effet que nombre des femmes du staff sont en couple avec d'autres bénévoles de l'association. Elles sont postées entre les deux amphithéâtres accueillant les joueureuse-s. Elles passent la majorité du tournoi fidèles à leur poste à assurer le service et l'intendance. Durant les temps morts elles discutent entre elles, à voix basses, et prennent rarement de nouvelles des matchs. Elles sont souriantes et aimables, veillent à la température du café et tâchent de donner aux joueureuse's des informations précises concernant l'heure de livraison des pizzas que tous attendent avec impatience. Elles se relaient quand viennent les heures les plus tardives. Tout comme le font les hôtesses d'accueil analysées par la sociologue Gabrielle Schütz (2006), elles prodiguent un accueil agréable et effectuent des tâches de type domestique (gestion de l'alimentation). Leur participation semble « reposer presque entièrement sur un savoir-être genré » (Schütz, $2006: 20)$. 
Illustration 2 et 3 : « Les filles du staff chargées de l'accueil du confort et de la promotion »
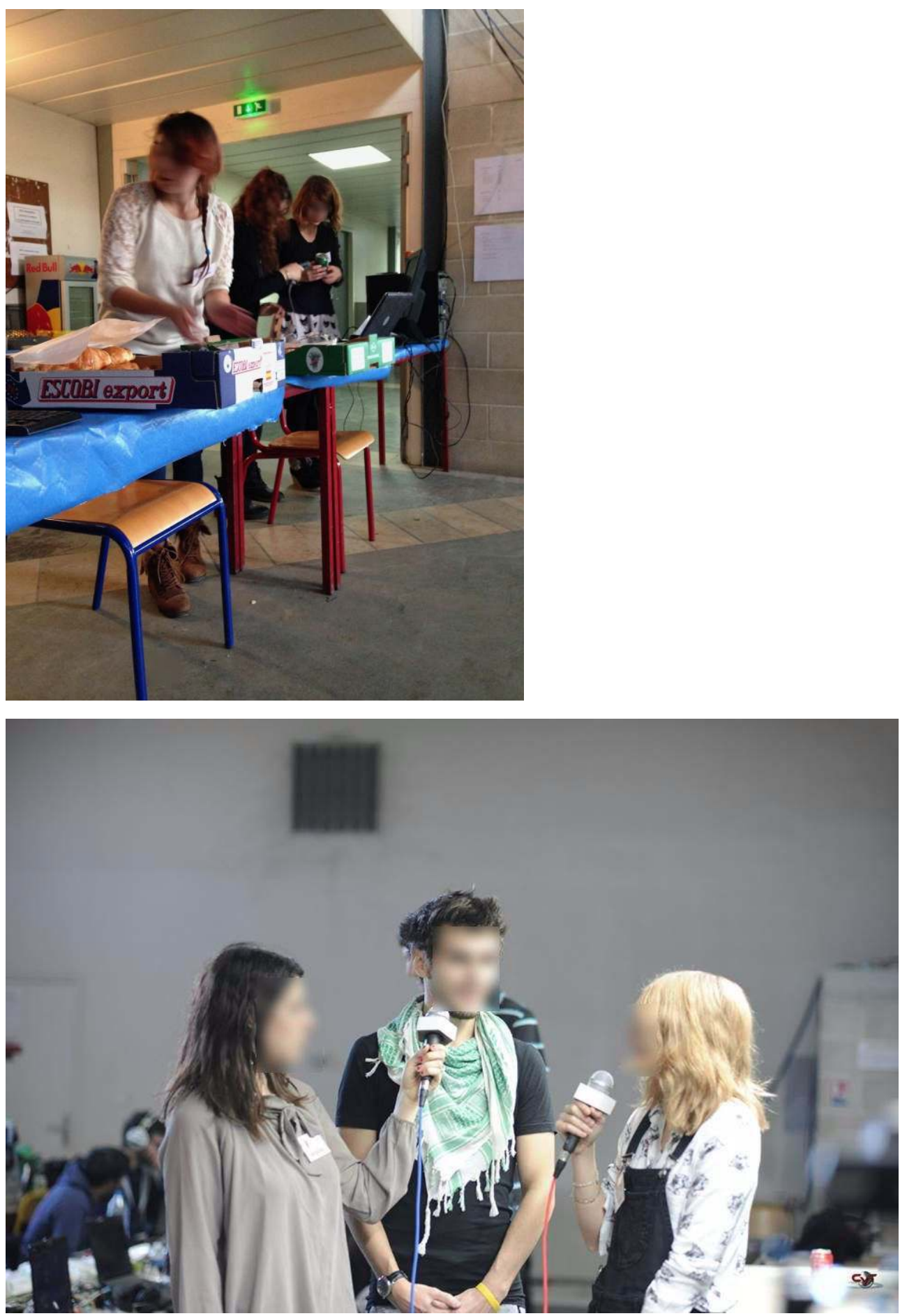

Source : Page Facebook de l'association «CYL », septembre et décembre 2015. URL : https:// www.facebook.com/ComputYourlan/photos_[consulté en décembre 2015].

La succession de nos observations laisse néanmoins apparaître quelques évolutions quant aux modes de participation des femmes bénévoles. Très discrètes et recluses dans l'espace restreint dédié à la vente lors des trois premières éditions, elles se voient confier des tâches plus diverses et moins inscrites dans la domesticité lors de nos dernières observations. En effet, certaines sont en charge d'interviews retransmises en direct 
(Youtube, page Facebook) ; elles recueillent les impressions des joueurs sur la compétition, loin des commentaires en direct des matchs effectués eux par des hommes. À la manière des hôtesses, les nouvelles tâches qui leur sont dévolues consistent, une fois encore, à la mise en scène et à la promotion principalement des joueurs. Elles ne pénètrent donc pas pour autant le domaine technique qui reste le domaine des hommes. Ces « filles du staff " semblent être des "supportrices discrètes » (Saouter, 1995) soutenant l'investissement de leur conjoint, en assurant la stratégie promotionnelle et une certaine attractivité de la LAN partie. Malgré la dimension fonctionnelle des rôles occupés, elles demeurent investies dans la vie du « boy's club » (Taylor, Witkowski, 2010). En effet, il est tout autant important de souligner que « les filles du staff », ne sont ni absentes ni dans des postures passives dans un univers de pratique masculine, elles participent à son bon fonctionnement. Toutefois, ce champs d'action reste cantonné exclusivement à des rôles de care et de représentation de genre aux fonctions « décoratives »(Schütz, 2006).

\section{Les joueuses : des formes de participation plus ambiguës}

Très minoritaires $(4 \%)$ le positionnement et le rôle des femmes participant au tournoi sont quant à eux beaucoup moins univoques et clairs. Les quelques femmes qui s'essayent à prendre directement part à la compétition semblent aux prises avec un ensemble d'injonctions dissonantes voire paradoxales.

Un premier constat s'impose : les joueuses sont toujours seules au sein de leur équipe. S'attarder sur la galerie des photos d'équipes réalisées lors de l'événement permet de constater combien le genre apparait, au sein de leurs équipes, comme un élément de qualification et de classement pertinent. En effet, sur les photos de groupe, les joueurs se répartissent selon deux catégories très claires que sont la taille et le genre. La seule joueuse de l'équipe se trouve, à quelques exceptions près, située au milieu de la photo, entourée par deux paires de joueurs ${ }^{13}$. 
Illustration 4,5 et 6 : Une place au centre
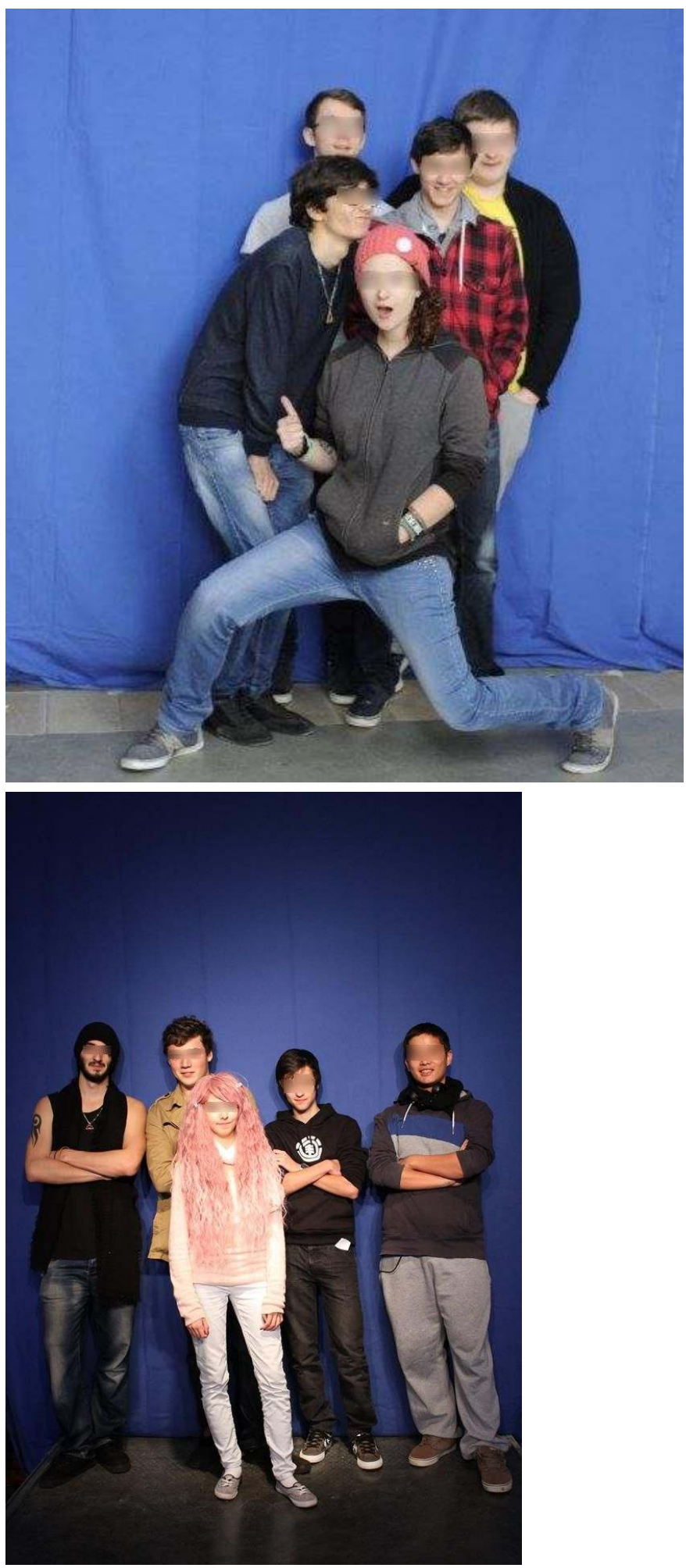


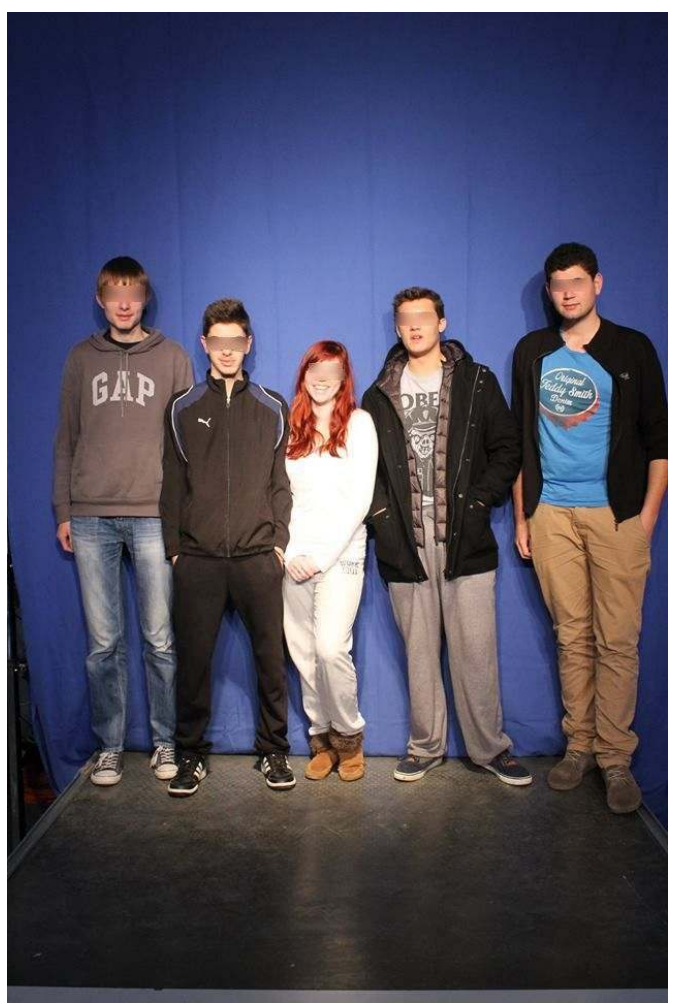

Source : Page Facebook de l'association «CYL», novembre 2014. URL : https://www.facebook.com/ ComputYourlan/photos_[consulté en novembre 2014].

Nous avons distingué deux façons d'incarner le rôle de joueuse, assez radicalement opposées et séparées par une barrière qui semble difficilement franchissable. Pas de demi-mesure en effet chez les joueuses " hyper féminines ", pas davantage chez les " garçons manqués ». Les premières sont maquillées et apprêtées, bien loin de la décontraction qu'affichent ostensiblement leurs camarades de jeu masculins. Le matériel qu'elles emportent est à leur image et peut, lui aussi, être « féminisé » telle cette joueuse disposant d'un casque rose ; une autre participante est quant à elle la seule, dans tout l'amphithéâtre, à disposer d'une tour de PC blanche quand tous les joueurs autour d'elle ont des tours noires. La sienne se trouve par ailleurs ornée d'un large autocollant représentant un personnage de manga féminin hypersexualisé. Ces joueuses sont, proportionnellement, bien moins nombreuses que ne le sont les petites amies de joueur nous y reviendrons - mais elles arborent, elles aussi, une féminité stéréotypée. Ce sont des femmes, dans un milieu majoritairement masculin, elles l'assument et semblent vouloir le rendre visible d'une certaine manière, sans afficher pour autant quelque forme de revendication.

Comme pour conquérir une place qui n'est pas tout à fait la leur et à laquelle elles ne pensent peut-être pas légitimement pouvoir prétendre, les secondes adoptent les codes vestimentaires des hommes joueurs. Sweat à capuche de couleur sombre et pantalons amples sont légion. Il s'agit semble-t-il pour ces joueuses de se " fondre dans la masse " des joueurs en adoptant leur apparence. Si certaines tendent alors à disparaître, d'autres en viennent parfois à afficher des comportements et attitudes plus virilisantes que les hommes eux-mêmes. Ainsi, Lomé (pseudo anonymisé) rote bruyamment et ne manque pas d'assortir son geste des commentaires potaches de rigueur. On pense ici à la " surenchère grossière " évoquée par Emmanuelle Zolesio qui explique combien intégrer un milieu d'hommes peut relever du " défi " pour des internes en chirurgie « sans cesse 
renvoyées à leur statut de femme (dominée) » et pour qui l'enjeu consiste à " s'extraire des rapports de séduction et de domination qu'essaient d'imposer leurs collègues hommes et de se positionner comme leurs égales. " (Zolesio, 2009, : 126) On voit ici combien leur présence dépend notamment de leur capacité à adopter des « comportements masculins » qui passe également par l'utilisation d'un « un vocabulaire ou un humour plutôt associé à la virilité » (Boussard, 2016: 60). Le comportement de Lomé n'est pas sans rappeler également les analyses d'Isabelle Collet dans les filaires d'informatiques. Celle-ci affirme en effet que " rire aux plaisanteries sexistes n'est pas seulement un subterfuge pour se faire accepter, mais aussi un moyen de se démarquer du groupe des "vraies" filles» (Mosconi \& Dahl-Lanotte, 2003; Collet, 2006). Elle parle également « d'élévation symbolique pour les garçons manqués qui quittent le groupe des dominés pour aller vers le groupe des dominants par nature inaccessible " (ibid. : 205). Pourtant, si Lomé - revendique même - certains codes et attitudes "masculins ", elle reste assignée à une place de femme dans le rôle qui lui est attribué au sein du collectif : largement passive, elle reste à l'écoute des directives des autres joueurs. De la même façon, nous constatons qu'elle adopte, dans le jeu, une attitude de soutien, déjà relevé par chez les joueuses de League of Legends (Rabindra, Taylor, Hogan, 2015), ou qu'elle se sacrifie pour le bien de l'équipe. Comme elle l'affirme, « ça me fait chier d'être focus mais ça permet à ma team de faire tellement de beaux trucs que je m'en fous ».

Ces mécanismes, révélateurs d'agencements de genre dans un univers dominé par les hommes, font écho enfin à l'analyse que propose Alice Aterianus-Owanga (2016) étudiant la place des femmes dans le rap gabonais. Cette dernière décrit en effet la façon dont les femmes négocient " différentes conceptions de la féminité » (Aterianus-Owanga, ibid.: 49). Certaines se définissent ou sont définies comme des "petits hommes » : une part de l'emprunt de la masculinité passe alors, à la manière des joueuses, par les vêtements ou encore un ensemble d'attitudes corporelles. L'auteure propose la catégorie de « femme garçonne » dont la production « constitue le produit d'un agencement sélectif et d'une adaptation localisée des modèles proposés » (ibid. : 51) pour offrir une alternative aux qualifications de " garçon manqué/manquant » (Mennesson \& Galissaire, 2004) ou encore celle du «troisième sexe » (Saladin D’Anglure, 2004). Si ces derniers sont mobilisés pour comprendre l'insertion de certaines femmes dans des espaces de pratique perçus comme masculin, ils ne rendent toutefois pas compte de la dimension pragmatique et localisée des agencements de genre. Ils sont, en LAN partie, primordiaux puisque, ici comme dans le milieu du rap, le fait de participer implique, quand on est une femme, de s'approprier certains des codes de la masculinité, contraintes qu'elles sont de naviguer et négocier leurs présences entre passivité des rôles occupés et performativité des attitudes (T.L. Taylor, 2012).

Dans l'univers de la chirurgie comme dans celui des compétitions publiques de jeu vidéo, les " manières étiquetées comme “ féminines" " (Zolesio, 2009: 123) sont stigmatisées et dépréciées. Dès lors, comme chez les chirurgiennes observées par Emmanuelle Zolesio dans son enquête (ibid.), être une bonne joueuse implique de se "masculiniser » et de se départir de ces façons d'être incompatibles avec la posture qu'appellent des activités culturellement considérées comme masculines, du fait notamment de leur technicité. Ces processus de "masculinisation " semblent particulièrement prégnants lors de la phase d'entrée dans le milieu où il s'agit pour les femmes de légitimer et de gagner leur place au sein de l'environnement masculin. Ceci peut expliquer le fait que certaines femmes 
(relativement peu nombreuses malgré tout) investissent ostensiblement certains signes visibles de féminité.

Isabel Boni-Le Goff décrit les opérations par lesquelles passe la construction de la légitimité de la figure d'expert pour les femmes (Boni-Le Goff, 2013). Dans le cas des femmes experts comme dans celui des joueuses, nous pouvons constater les différents passages, transgressions, négociations et compromis que les femmes doivent accomplir pour construire, localement et au cours des interactions, une place au sein d'un environnement social où règnent les comportements, us et coutumes virils. Cette auteure évoque par ailleurs les différentes formes d'injonction qui pèsent sur les femmes au niveau d'une apparence physique toujours problématisée : oscillant entre les deux figures tout aussi stigmatisées et stigmatisantes que peuvent être les femmes " androgynes » ou les « bimbo ", le comportement le plus prudent consiste alors à revêtir l'apparence de « la madone » et ainsi, en prenant garde de ne pas se faire remarquer, se «fondre » dans un décor et un univers masculin "gris et neutre ", référence à laquelle il convient de se conformer. Ce «stratagème (...) permet d'apporter une réponse médiane à la double question de la sexualisation vestimentaire (pas d'hyper sexualisation, pas de transgression vestimentaire des frontières de genre) et du dosage (porter un masque... qui ne se voit pas trop.) »(Boni-Le Goff, $2013:$ 459)

Pourtant, quelles que soient ces stratagèmes que développent et mettent en place les femmes, la position qu'elles adoptent autant que les paradoxes fréquents qu'offrent à voir leurs différents modes de participation laissent penser qu'elles ne peuvent parvenir à accéder, dans ces espaces, au statut de joueuses à part entière. En effet, "l'injonction fondamentale est de marquer sa différence sexuelle : ne pas oublier qu'on reste une femme dans un collectif d'hommes, c'est-à-dire ne pas croire qu'une collègue égale un collègue " (Cromer et Lemaire, : 73). Certainement le fait que, même lorsqu'elles font partie des équipes prenant part à la compétition, elles continuent à assumer les tâches domestiques et de soutien traditionnellement dévolues aux femmes confirme-t-il cette impression qu'aucun de leurs efforts pour faire leurs les codes masculins ou, au contraire, pour se distinguer des hommes, ne suffit à résoudre ce qui apparaît comme une incompatibilité fondamentale entre leur sexe et l'ordre cœur - technique - d'activité en cours et les qualités qu'il requiert. Le féminin joue le rôle de figure repoussoir dont il s'agit de se distinguer ce qui semble, a priori, enfermer les femmes dans une position d' outsider (Rabindra, Taylor, Hogan, 2015).

\section{Les petites amies}

41 La figure des « petites amies » est quantitativement majoritaire. Figure la plus éloignée de l'action directe de jeu, les " petites amies » affichent une posture majoritairement passive. "Petites amies » est ici employé dans un sens conjugal et non contractualisé institutionnellement, et sous-tend des formes de publicisation du lien comparables à celles des femmes présentes dans les tribunes lors des matchs de rugby analysées par Anne Saouter (2010). Leurs positions et façons de faire peuvent également être rapprochées de celles des "groupies" (Saouter, 1995; Turbé, 2016), des hôtesses évoquées plus haut (Schütz, 2006) ou encore " des épouses invitées à une certaine discrétion » (Saouter, 1995) toutes examinées dans le cadre de pratiques culturelles et ou sportives dites masculines. 
42 À la différence des « filles du staff », les « petites amies » sont presque totalement dédiées aux tâches d'intendance, dans le prolongement de la sphère domestique. Elles transportent l'ensemble des items nécessaires à assurer le confort de leur conjoint pendant les longues heures que va durer le tournoi. Cigarettes, snacks, monnaie, chargeurs de téléphone sont prêts et à disposition si apparaît le besoin. À la manière du linge des rugbymen (Saouter, 1995), elles semblent avoir anticipé toutes les situations afin d'éviter les pertes de temps. En cas d'épuisement des stocks ou de commande particulière, elles se déplacent au sein des amphithéâtres et hors des murs de la LAN dans le but d'approvisionner leur conjoint autant que les membres de son équipe.

Deux femmes arrivent avec des cafés pour tous les joueurs (CYL, JSB, avril 2014).

$16 \mathrm{~h} 20$ : Une femme [avec] deux poches de Macdonald pleines à craquer » (CYL, JSB, avril 2014).

43 À la manière des hôtesses, elles apparaissent comme les gestionnaires des tâches de type domestique (Schütz, 2006). Parfois, avant la compétition ou quand l'intensité du tournoi laisse entrevoir les premiers signes de fatigue, elles s'improvisent masseuses pour prévenir ou soulager les tensions accumulées dans les corps.

Une femme arrive, elle installe le nécessaire pour son conjoint/joueur : une bouteille de coca, un paquet de gâteaux ; elle met son téléphone portable en charge.

Il s'installe et elle le masse (épaules, tête) (CYL, JSB, avril 2014).

44 Les pratiques des " petites amies » - gestion/attention aux provisions, prévention et soins accordés au bien-être corporel - sont, elles aussi, largement inscrites dans le registre du care (Cresson, Gadrey, 2004), déjà mentionnées dans la figure précédente (filles du staff et joueuses). Elles apparaissent également comme le reflet des formes genrées de la division conjugale des tâches et activités. La façon dont les "petites amies » occupent l'espace est corrélée aux missions qui sont les leurs. Durant des heures, elles restent assises, à une place spécifique qui ne varie que peu, en se fondant littéralement dans le dos de leur petit ami joueur. Elles font corps avec lui. Loin d'être une métaphore, les corps montrent non pas une " dimension discrète ", à la manière des conjointes dans le rugby qui verbalisent leurs encouragements et attendent les joueurs entre elles après le match (Saouter, 1995), mais plutôt une « fusion directe » doublé d'un dévouement continu.

Illustration 7 et 8 : Les petites amies des joueurs les regardent jouer, assises derrière eux.






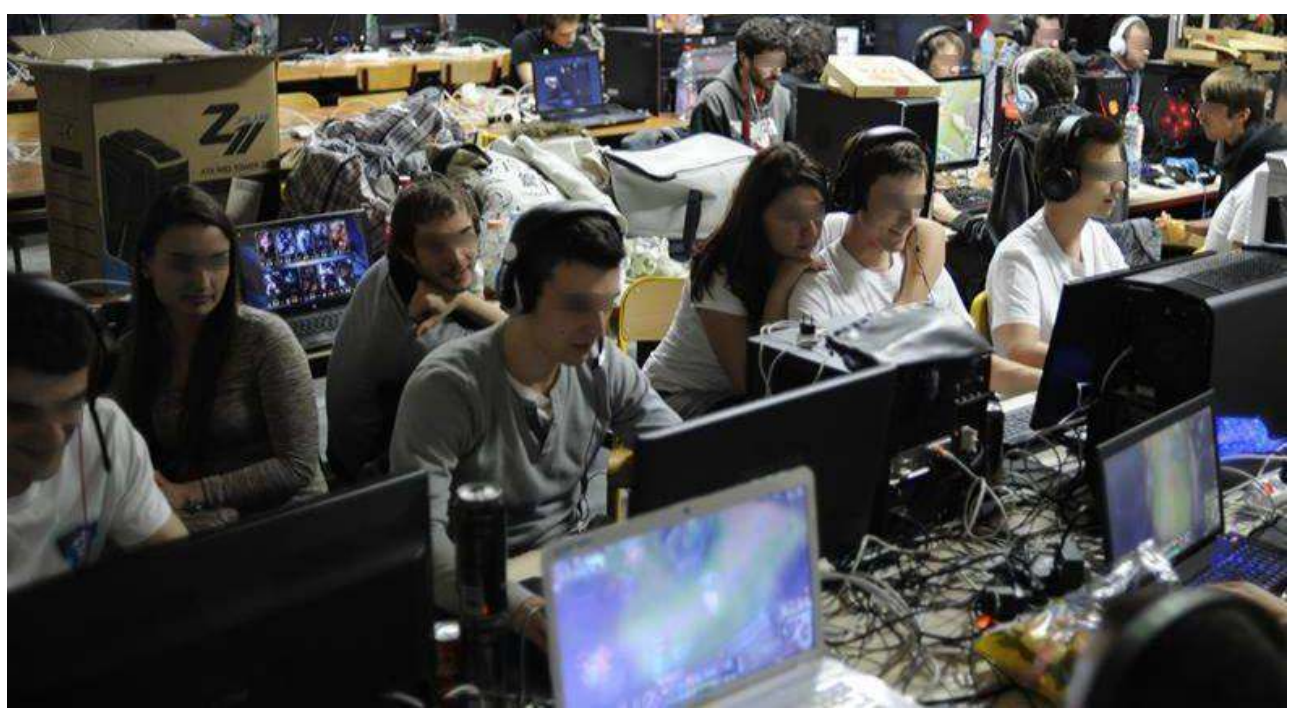

Source : Page Facebook de l'association «CYL », décembre 2015. URL : https://www.facebook.com/ ComputYourlan/photos_[consulté en décembre 2015].

14h38: Comptage des copines. J'en compte 10, 10, 11. Une toute tordue à la manière d'une koala » (LAN CYL, JSB, avril 2014).

Salle de visionnage, 1h40: Groupe 3, arrivent 5 hommes, 2 femmes (petites amies), elles s'assoient derrière le groupe.

1 h50 : groupe 3 part. Les deux femmes restent. [Silence]. Une des deux femmes, à l'autre :

- «tu crois qu'on devrait y aller?»

- « Oui, on va les suivre» (LAN, JSB, ComUniTIC, mai 2013).

$\mathrm{Si}$ « les groupies » ou " fans " décrites dans la littérature montrent un investissement passionnel et expert dans les pratiques culturelles, les « petites amies ", elles, demeurent passives et d'une patience à toute épreuve. La discrétion est de mise ; elle constitue précisément une des qualités de la «bonne copine » (Clair, 2012). Toujours présentes, elles ne regardent pourtant que très rarement les écrans de jeu. Elles semblent subir le rythme du week-end et de la compétition. Les temps de pause entre les parties, lors desquels les joueurs quittent souvent les amphithéâtres le temps d'une cigarette, constituent pour elles une des rares occasions de sociabilité. Quand elles suivent le mouvement de l'équipe, il peut leur arriver de participer discrètement aux échanges ; les joueurs, exaltés suite à la fin de la partie, occupent généralement la majeure partie des interactions. Si elles décident de rester assises, ce moment peut alors être l'occasion d'échanges entre supportrices. En dehors de ces interactions minimes le silence est de rigueur. Les joueurs, à l'image des mécaniciens décrits par Sophie Denave et Fanny Renard (2015), se montrent peu disponibles envers leur compagne. À la différence d'autres conjointes décrites dans des univers masculins (Denave, Renard, 2015 ; Saouter, 1995), les petites amies demeurent néanmoins présentes au cœur de l'action et ont accès à l'ensemble des espaces des " petits amis »; nous voyons pourtant qu'elles ne font de cette « liberté » de mouvement qu'un usage très limité.

\section{Les femmes comme objet de hiérarchisation interne au groupe des hommes}

Outre le soutien concret qu'elles apportent, les petites amies occupent une place importante dans l'ordre symbolique de l'événement. De toute évidence, dans la photo ci- 
dessous, la supportrice participe, au même titre que les T-shirts à l'effigie de leur équipe que porte chaque joueur, du prestige des membres (Illustration 9) qu'elle est venue soutenir et dont elle contribue à accroître, de par son élégance et son apparence physique soignée, le capital symbolique. Par leur simple présence, les femmes permettent aux membres de cette équipe de se distinguer du reste des joueurs. Elles jouent alors le rôle d'hôtesses puisque, outre leur intérêt fonctionnel, elles sont de véritables «objets esthétiques " qui «entretiennent ainsi le capital symbolique " des joueurs qu'elles accompagnent « en réaffirmant leurs hiérarchies sociales internes et externes. » (Schütz, $2006,: 149)$

«La fille très sexy » est visiblement la copine de J3 de la team E3. Elle lui caresse le dos et fait un commentaire sur son t-shirt. Pendant qu'il fait la queue elle vient lui faire une caresse. La fille en face d'elle est sûrement une copine d'un des membres de l'équipe j'imagine. Les deux filles discutent avec deux membres de l'équipe des E3. Ils parlent de la vie de la team et des derniers événements, la dispute de la veille. Ils rient, les filles aussi. Ils discutent du jeu sans employer semble-t-il trop de termes techniques. Les garçons vont fumer dehors. « La fille sexy » retourne dans la queue faire des caresses dans le dos de J3. Elle fait démonstration. Elle est la plus jolie et la mieux habillée ; son copain porte le t-shirt de son équipe (LAN CYL, JC, Novembre 2014).

Illustration 9 : Échange dans le hall entre un joueur et deux femmes accompagnant son équipe

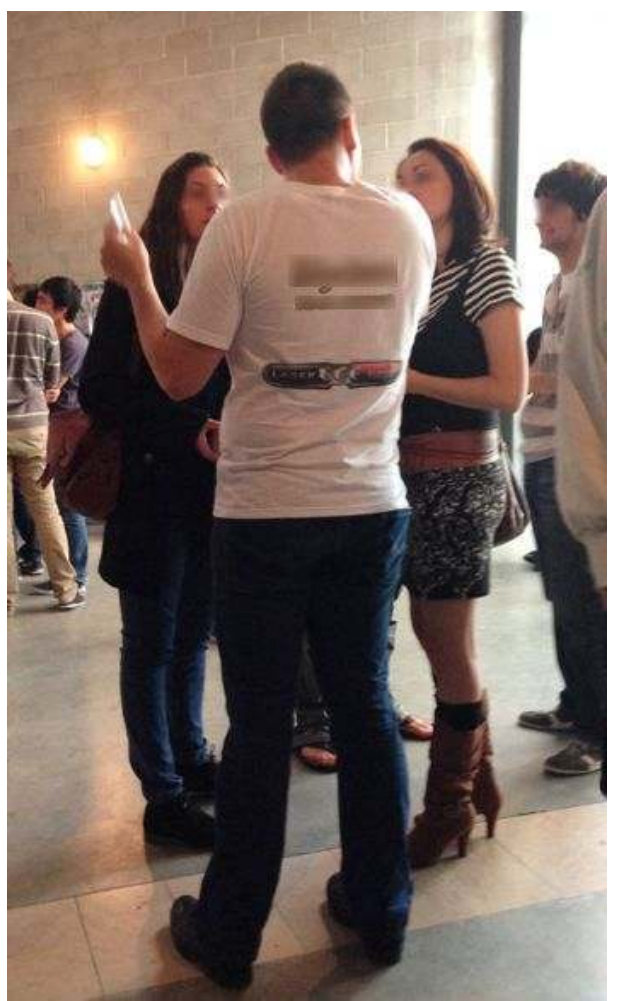

Source : Photographie réalisée par l'enquêteur, CYL 2014.

La conjugalité hétérosexuelle est par ailleurs la norme et semble revêtir, elle aussi, un véritable enjeu de démonstration pour les joueurs. Cette mise en scène conjugale peut être analysée comme une réaction à la figure stéréotypée et stigmatisante du geek solitaire et incapable de séduire une femme. Faire démonstration de sa réussite semble donc passer tout autant par des actions « dans le jeu » ou avec la machine grâce à laquelle il est possible de montrer son habilité individuel de sportif (T.L Taylor, 2012) que « hors 
du jeu » via l'affichage de sa conjugalité. Cette importance de la mise en couple a déjà été révélée par Nicolas Taylor (2015) dans son enquête sur les joueurs professionnels de sport électronique qui insistent dans les entretiens sur l'équilibre de leur vie romantique. Elle se donne ici publiquement à voir avec des " petites amies " assurant "l'image publique " (Schütz, 2006) du couple. Le « ballet » des couples, témoigne, au-delà de la description de la division fonctionnelle sexuée des places, de la façon dont le genre se déploie. Il s'agit pour les joueurs de se montrer en couple pour se distinguer et se faire valoir auprès de ceux qui, contrairement à eux, ne sont pas parvenus à allier pratique intensive des jeux vidéo et relation conjugale.

Dans la salle de projection un des 3 mecs qui est venu avec sa copine est assis au milieu de la salle. Sa copine vient l'embrasser en restant debout devant tous les spectateurs, précisément placée entre eux et l'écran qui diffuse le match (LAN, JC, ComUniTIC, mai 2013).

En effet, les spectatrices ne semblent pas être là par hasard et/ou pour seulement leur plaisir personnel comme nous l'avons montré plus haut au travers de la passivité qu'elles témoignent à l'égard du jeu et de la compétition. Les stigmates de la figure du Keyboard jockey (séducteur du clavier) décrit dans les travaux de Mélanie Gourarier (2014, 2017) "passant beaucoup trop de temps devant leur ordinateur au détriment des sociabilités dans leur "vrai vie" " (ibid., 2014, p.46) imprègnent l'image de soi que les uns et les autres tentent de renvoyer et conditionnent les modalités effectives de la présence des petites amies de joueurs en LAN parties. Cette volonté de définition en opposition pourrait être résumée par ce verbatim emprunté aux travaux de Peyron (2013) sur la culture geek : " je me définis comme un geek mais qui baise " (Théo, 28 ans). Ces passionnés d'informatique et de jeux vidéo, confinés pendant longtemps au bas de la hiérarchie des masculinités (T.L. Taylor, 2012), trouvent dans ces évènements des espaces et des occasions de s'élever publiquement au sein de la communauté des hommes. Nous retrouvons une tension en LAN parties lors desquelles nombre des éléments de la culture geek (savoirs experts et vocabulaire) fédèrent visiblement les joueurs chez lesquels transparaît pourtant également une volonté d'être assimilés au groupe des hommes " comme les autres ", qui implique la mise en scène de la virilité et, quand cela est possible, de la conjugalité. Ainsi, les rapports de genre apparaissent comme une forme de politisation de « la revanche des geek» (Peyron, 2013) et un moyen pour les joueurs de se réapproprier les figures stigmatisantes, au sein même de la communauté des joueurs, du geek et du nolife (Gerber, 2015). Il apparaît bien, à l'évidence, que « le terme sert de figure repoussoir, servant à qualifier quelqu'un (ou une partie de soi) dont on cherche à se distancier, parce que le nolife passe un nombre d'heures excessif à jouer et n'a pas d'autres intérêts ni responsabilités; il n'a en somme pas de "vraie" vie " (Gerber, 2015). Être en couple constitue ici un moyen de faire la démonstration qu'il est possible de (bien) jouer aux jeux-vidéos tout en ayant une vie sociale et sentimentale satisfaisantes.

\section{Triptyque du care}

Nos observations s'opposent à ce que certains présentent comme une évolution de la place et du statut des femmes en LAN parties (Taylor et Witkowski, 2010). Nous rejoignons au contraire les analyses de Bryce et Rutter $(2002,2003)$ puisque, dans notre cas comme dans le leur, les femmes sont encore assez largement cantonnées à des rôles de soutien et d'assistance, passives ou soumises à l'approbation des hommes. Quand bien même ces rôles et les façons de les incarner diffèrent assez fortement les uns des autres, et même si 
les frontières entre chacun d'entre eux semblent infranchissables, toutes partagent en effet un attribut commun : en tant que femmes elles sont en charge des aspects logistiques. Quand les équipes comptent une femme dans leurs rangs - ce qui reste rare -, elles demeurent majoritairement silencieuses (Rabindra, Taylor, Hogan, 2015) et sont celles qui transportent et organisent les vivres pour l'ensemble de l'équipe. Les petites amies assument elles aussi ces fonctions qui vont de soi pour les filles du staff dont c'est précisément la fonction. Quand bien même la raison de leur présence diffère, les femmes en LAN parties s'occupent dans leur quasi-totalité du confort des joueurs. "Les petites amies » sont sollicitées pour aller chercher tout ce qui pourrait manquer aux équipes tout au long de leur participation (nourriture, cigarettes et matériel oublié). Elles sont également, à travers leurs sacs à main, les gardiennes des effets personnels des joueurs. Outre les aspects matériels et alimentaires, le confort de leur conjoint passe parfois par des massages à la manière des soins apportés aux sportifs de haut niveau. Les joueuses s'occupent elles aussi de la gestion des repas et anticipent le grignotage de leurs coéquipiers. Certaines filles du staff vont au-delà de leurs attributions de bénévoles et proposent aux joueurs des pâtisseries faites maison. À l'inverse, personne ne semble se soucier de leur bien-être. Les LAN parties peuvent, dans une certaine mesure, être considérées comme une extension de la sphère domestique au sein de laquelle les femmes restent souvent chargées en priorité des tâches ménagères (Guillaumin, 1978). Largement naturalisé, ce rôle ne semble nullement ici être remis en question. La présence plus importante des femmes n'entraîne visiblement pas davantage de résistance ni de remise en cause de l'hégémonie masculine de ces arènes vidéoludiques. Ce triptyque s'articule autour de fonctions de soutien et de soin qui se déploient à l'intérieur et à l'extérieur du jeu les femmes se voient chargées d'accomplir le « sale boulot " (ibid.) et d'assumer le rôle de «maitresse de maison"; rôle qui se superpose aux autres activités quand il ne constitue pas déjà en soi la raison d'être de leur présence.

\section{Conclusion}

Sans dire que les LAN parties mettent en scène un univers social sclérosé autour des assignations traditionnelles de genre et sans rejeter l'idée d'un possible renouveau de ces rapports, on constate pourtant combien certaines assignations de genre traditionnelles demeurent, au sein des jeunes générations de joueureuse's, solidement ancrées et prégnantes. Ces événements apparaissent tout à la fois comme une occasion de mise en scène, de réassurance et de reproduction des attributs de genre que la technique vient sceller.

51 Qu'ils y adhèrent fortement ou non, les hommes se trouvent pris dans une logique collective qui valorise une identité de joueur viril, puissant et performant qui se construit, dans le discours et le verbe notamment par l'usage d'un vocabulaire viril et en opposition aux figures repoussoir que sont les femmes ou les homosexuels (Clair, 2012). Les femmes quant à elles se voient cantonnées à un nombre limité de rôles qu'il leur est offert de jouer. De rôles entièrement dédiés au soutien de leurs pairs masculins chez les membres du staff ou les compagnes de joueurs, leur place devient plus ambiguë, complexe, voire paradoxale dès lors qu'elles se saisissent des claviers et souris pour prendre activement part à la compétition. Pourtant, même joueuses, le poids des assignations continue de peser lourdement sur les formes possibles de participation. Ces dernières semblent en effet être confrontées à une tension entre des injonctions 
contradictoires. Elles sont des femmes sur lesquelles pèsent nombre d'impératifs genrés ; elles sont aussi des joueuses et sont donc, à ce titre, invitées à endosser des attributs virils. Les manières individuelles de composer avec ces formes de participation paradoxales diffèrent alors d'une joueuse à l'autre quand bien même celles-ci, comme l'ensemble des femmes présentes, se doivent d'assumer les fonctions du care.

Éminemment masculine, la culture ici mise en jeu et en scène oblige les femmes à prendre position entre un rôle hyper féminisé ou virilisant, l'un et l'autre maintenant ces joueuses dans une position de soutien dont il semble encore difficile de se défaire. Il serait dès lors pertinent d'interroger, par le biais d'entretien et/ou d'observations spécifiques, les motivations des joueuses et leurs modes d'intégration ainsi que celles des femmes spectatrices - les plus présentes numériquement dans ces espaces - afin de questionner plus avant cette forme de participation et cette apparente passivité.

En affichant une «image rassurante de la femme », les femmes présentes en LAN parties participent à la « réassurance des identités hétérosexuées " (Schütz, 2006 : 149). Loin de remettre en cause la hiérarchie des sexes qui se dessine autour de la technique et de la maîtrise des outils, la présence des femmes permet au contraire aux hommes de se décharger des tâches domestiques, d'accroître leur capital symbolique et de se distinguer d'autres participants ou d'autres joueurs moins dotés tout en reproduisant une domination masculine séculaire. Les femmes permettent en effet aux hommes d'échapper à une assignation négative qui menace le joueur expert et fortement investi dans le jeu en lui offrant l'occasion de faire publiquement démonstration que cet état de fait ne l'empêche nullement de jouir d'un autre des attributs de la masculinité souhaitée et souhaitable que représente le pouvoir de séduction.

De par leur présence, loin d'une dynamique d'inclusion, elles semblent contribuer, de manière relativement paradoxale, à la "fabrique du genre » (Schütz, 2006: 149) qui les place, de fait, en situation de subordination et d'appropriation. En effet, par les tâches et les rôles qu'elles campent et les logiques de performances hétéronormatives qu'elles participent à (re)produire, leurs présences semblent être «au service de» (N. Taylor, 2009) et instrumentalisées dans une volonté de renégociation de la masculinité des joueurs. Celle-ci se donne alors à voir de manière processuelle au travers d'une configuration de pratiques (Connell, 2014 ; T.L Taylor, 2012) ici celle des jeux vidéo. Ainsi, cette renégociation de la masculinité par le biais des pratiques prend toute sa dimension en LAN partie avec des logiques d'habilités et de performances conjugales situées face à une audience dans l'espace public. Il apparait comme un lieu de valorisation de la masculinité hégémonique (Gourarier, 2014).

Au sein de ces espaces, la négociation de la masculinité des joueurs s'inscrit dans un double rapport de " complicité » (Connell, 2014) et d'exclusion (T.L Taylor, 2012). Complicité dans la mesure où les joueurs profitent des dividendes de la domination masculine passant en grande partie par la directe « subordination des femmes » (Denave, Renard, 2015). Exclusion également des formes hégémoniques de la masculinité dont souffrent les joueurs affublés des stigmates de la culture geek. Les LAN parties apparaissent alors comme des espaces publics et ludiques de pratiques de la masculinité par et pour les joueurs ainsi que des lieux de passage - sous contrôle - de la figure du " looser » à celle du mâle dominant (Gourarier, 2017). 


\section{BIBLIOGRAPHIE}

ATERIANUS-OWANGA Alice (2016). « Tu t'en es pris à la mauvaise go! » Transgresser les normes de genre sur les scènes rap du Gabon », Ethnologie française, 161 (1), pp. 45-58.

AURAY Nicolas (2002). "Sociabilité informatique et différence sexuelle », in Chabaud-Rychter Danielle \& Gardey Delphine (dir.), L'engendrement des choses, des hommes, des femmes et des techniques, Éditions des Archives Contemporaines, pp. 123-148.

AURAY Nicolas (2007). « Miroir et balai de sorcière », in Beau Franck (al.), Culture d'univers : jeux en réseau, mondes virtuels, le nouvel âge de la société numérique, Broché, pp. 100-108.

BAUDELOT Christian \& ESTABLET Roger (2007). Quoi de neuf chez les filles? Entre stéréotypes et libertés. Paris, Nathan.

BERRY Vincent (2011). «Sociologie des MMORPG et profils de joueurs : pour une théorie sociale de l'activité (vidéo)ludique en ligne », Revue des Sciences Sociales, 45, pp. 78-85.

BERRY Vincent (2012). L'expérience virtuelle : Jouer, vivre, apprendre dans un jeu vidéo. Rennes, PU Rennes.

BERTRAND Julien, COURT Martine, MENNESSON Christine \& ZABBAN Vinciane (2015). « Introduction. Socialisations masculines, de l'enfance à l'âge adulte », Terrains \& travaux, 27 (2), pp. 5-19.

BESOMBES Nicolas (2016). « Les jeux vidéo compétitifs au prisme des jeux sportifs : du sport au sport électronique », Sciences du jeu, 5 [en ligne], URL : http://journals.openedition.org/sdj/612.

BOUSSARD Valérie (2016). «Celles qui survivent : dispositions improbables des dirigeantes dans la finance », Travail, genre et sociétés, 35 (1), pp. 47-65.

BOUTET Manuel (2012). « Jouer aux jeux vidéo avec style. Pour une ethnographie des sociabilités vidéoludiques ", Réseaux, 173-174 (3), pp. 207-234.

BRYCE Jo \& RUTTER Jason (2002). « Killing like a girl. Gendered Gaming and Girl Gamers's Visibility », in Mäyrä Frans Proceedings of Computer Games and Digital Cultures Conference, Tampere, Tampere University University Press, pp.243-255.

BRYCE Jo \& RUTTER Jason (2003). « The Gendering of Computer Gaming : Expérience and Space », in Fleming S \& JONES, I, (dir.) Leisure Culture : Investigations, Sport, Media and Technology, Leisure Studies Association, pp.3-22.

CLAIR Isabelle (2012). « Le pédé, la pute et l'ordre hétérosexuel », Agora débats/jeunesses, 60 (1), pp. 67-78.

COAVOUX Samuel \& GERBER David (2016). « Les pratiques ludiques des adultes entre affinités électives et sociabilités familiales », Sociologie, 7 (2), pp. 133-152.

COLLET Isabelle (2006). L'informatique a-t-elle un sexe ? Hackers, mythes et réalités, Paris, L'Harmattan. CONNELL Raewyn (2014). Masculinités enjeux sociaux de l'hégémonie. Paris, Amsterdam.

CRESSON Geneviève \& GADREY Nicole (2004). «Entre famille et métier : le travail du care », Nouvelles Questions Féministes, 23 (3), pp. 26-41. 
CROMER Sylvie \& LEMAIRE Dominique (2007). « L'affrontement des sexes en milieu de travail non mixte, observatoire du système de genre », Cahiers du Genre, 42 (1), pp. 61-78.

D'ANGLURE Bernard Saladin (2004). « Le « troisième » sexe social des Inuit », Diogène, 208 (4), pp. 157-168.

DENAVE Sophie \& RENARD Fanny (2015). « Aspirants mécaniciens, aspirants coiffeurs », Terrains \& travaux, 27 (2), pp. 59-77.

DODIER Nicolas (1993). « Les appuis conventionnels de l'action. Eléments de pragmatique sociologique », Réseaux, 62 (6), pp. 63-85.

DONNAT Olivier (2009). Les pratiques culturelles des Français à l'ère numérique. Paris, La Découverte. DUCROCQ Samuelle (2011). Les tribus ludiques du «LAN party» : perspectives d'apprentissage et de socialisation en contexte de compétition de jeux vidéo en réseau local, Thèse de doctorat, Université du Québec à Montréal, Montréal.

DURET Pascal (2009). Sociologie de la compétition: Sociologies contemporaines. Paris, Armand Colin. FINE Gary Alan (2015). Players and Pawns. How Chess Builds Community and Culture, Chicago: University of Chicago Press.

GERBER David (2015). « Le jeu vidéo comme pratique discréditable. Enquête sur les efforts de légitimation ordinaires des joueurs ", RESET. Recherches en sciences sociales sur Internet, 4, [en ligne]. URL : https://journals.openedition.org/reset/452.

GOFF Isabel Boni-Le (2013). Le sexe de l'expert. Régimes de genre et dynamique des inégalités dans l'espace du conseil en management, Thèse de doctorat, Ecole des Hautes Etudes en Sciences Sociales, Paris.

GOFFMAN Erving (2004). « Le déploiement du genre », Terrain. Anthropologie \& sciences humaines, 42, pp. 109-128.

GOURARIER Mélanie (2014). « Le (mauvais) genre de l'Internet. Séducteurs des rues/séducteurs de la Toile », Hermès, 69 (2), pp. 45-49.

GOURARIER, Mélanie (2017). Alpha mâle. Séduire les femmes pour s'apprécier entre hommes, Paris, Le Seuil.

GUILLAumin Colette (1978). « Pratique du pouvoir et idée de Nature (1) L'appropriation des femmes ", Questions Féministes, 2, pp. 5-30.

LAHIRE Bernard (2001). « Héritages sexués : incorporation des habitudes », in Bloss Thierry La dialectique des rapports hommes-femmes, Paris, Presses Universitaire de France, pp.9-25.

LEGoUT Marie-Christine (2003). « Modes de régulation des pratiques ludiques en salle de jeux réseau », Les Cahiers du numérique, Vol. 4 (2), pp. 135-148.

LIGNON, Fanny (dir.) (2015). Genre et jeux vidéo. Toulouse, Presses universitaire du Midi.

MENNESSON Christine \& GALISSAIRE Romain (2004). « Les femmes guides de haute montagne : modes de socialisation et identités sexuées », Recherches féministes, 17 (1), pp. 111-141.

MOSCONI Nicole \& DAHL-LANOTTE Rosine (2003). « C'est technique, est-ce pour elles ? ", Travail, genre et sociétés, 9 (1), pp. 71-90.

PEYRON David (2013). Culture geek. Limoges, FYP éditions. 
RABINDRA A Ratan Robby, TAYLOR Nicholas, HOGAN Jameson, KENNEDY Tracy, \& WILIIAMS Dmitri. (2015). «Stand by Your Man: An Examination of Gender Disparity in League of Legends », Games and Culture, 10 (5), pp.438-462.

SAOUTER Anne (1995). « La maman et la putain. Les hommes, les femmes et le rugby », Terrain. Anthropologie \& sciences humaines, 25, pp. 13-24.

SAOUTER Anne (2010). « Pratiques sportives et représentations du corps : consécration de l'éternel masculin », Empan, 79, pp. 105-110.

SCHMOLL Patrick (2003). «LE DÉBAT. Les jeux vidéo violents : un espace de médiation », Cultures en mouvement, 60 , pp. $46-49$.

schÜTZ Gabrielle (2006). « Hôtesse d'accueil », Terrains \& travaux, 10 (1), pp. 137-156.

TABET PAOLA (1998). La construction sociale de l'inégalité des sexes. Des outils et des armes, Paris/ Montréal, L'Harmattan.

TAYLOR Nicholas (2016). «Play to the camera: Video ethnography, spectatorship, and e-sports ", Convergence, 22 (2), pp. 115-130.

TAYLOR T.L (2009). Play Between Worlds. Exploring Online Game Culture. Cambridge, MIT Press.

TAYLOR T.L (2015). Raising the Stakes - E-Sports and the Professionalization of Computer Gaming.

Cambridge, MIT Press.

TAYLOR T.L \& WITKOWSKI Emma (2010). « This Is How We Play It: What a Mega-LAN Can Teach Us About Games », Proceedings of the Fifth International Conference on the Foundations of Digital Games, FDG'10, pp. 195-202.

TEBOUL Jeanne (2015). « Combattre et parader », Terrains \& travaux, 27 (2), pp. 99-115.

TRICLOT Mathieu (2015). «Où passe le genre ? Les jeux vidéo au prisme des théories féministes du cinéma », in Lignon Fanny (dir.) Genre et jeux vidéo, Toulouse, Presses universitaires du Midi, pp.19-36.

TURBÉ Sophie (2016). « Puissance, force et musique metal Quand les filles s'approprient les codes de la masculinité », Ethnologie française, 161 (1), pp. 93-102.

WELZER-LANG Daniel (1995). « Les transgressions sociales des définitions de la masculinité », in EPHESIA (dir.), La place des femmes. Les enjeux de l'identité et de l'égalité au regard des sciences sociales, Paris La Découverte, pp. 447-452.

WENDLING Thierry (2002). Ethnologie des joueurs d'échecs. Paris, Presses universitaires de France. WEST Candace \& ZIMMERMAN Don H. (1987). « Doing Gender », Gender and Society, 1 (2), pp. 125-151. ZABBAN Vinciane (2007). « Le jeu des médiations au service de la mise en tension des « univers virtuels » ", Réseaux, 143 (4), pp. 45-79.

ZABBAN Vinciane (2009). « Hors jeu? », Terrains \& travaux, 15 (1), pp. 81-104.

ZABBAN Vinciane (2011). « Ceci est un monde » Le partage des jeux en ligne : conceptions, techniques et pratiques, Thèse de doctorat, Paris, Université Paris-Est.

zOLESIO Emmanuelle (2012). Chirurgiens au féminin ? Des femmes dans un métier d'hommes, Rennes, Presses universitaires de Rennes. 
zoLESIO Emmanuelle (2009). «"Chirurgiennes de garde" et humour "chirurgical". Posture

féminine de surenchère dans l'humour sexuel et scatologique ", ¿ Interrogations ?, 8, [en ligne].

URL : http://www.revue-interrogations.org/Chirurchiennes-de-garde-et-humour.

\section{NOTES}

1. Syndicat National du Jeu Video voir baromètre annuel du jeu vidéo en France 2017 où les femmes représentent 14, 4\% des effectifs dans les studios de production, consulté le 25.12.2018, URL : http://snjv.org/wpcontent/uploads/2017/11/SNJV_barometre_2017_18.pdf.

2. Dans la suite du texte, le nom du jeu sera abrégé, tels que le font de la plupart des joueur.euse's, avec l'acronyme « LoL »

3. Abréviation anglaise de Multiplayer Online Battle Arena: arène de bataille en ligne multijoueureuse.

4. Lorsque des extraits d'observation sont présentés par la suite, le nom de la personne ayant réalisé l'observation est indiqué par les initiales : «jc » pour Johann Chaulet et « jsb » pour Jessica Soler-Benonie.

5. Communauté des universitaires autour des usages des technologies de l'information et de la communication, ce collectif entend favoriser les rapprochements et échanges entre chercheur·e's, usager·ère's et concepteur·trice's d'outils numériques.

6. Composé de deux pages de questions fermées (afin de faciliter les réponses), ce questionnaire a été distribué lors de la phase d'inscription matinale au tournois.

7. Lors de la passation du questionnaire (réalisée lors du troisième weekend de tournoi) les raisons de notre venue ont clairement été mise à jour, sans que nous ne puissions noter de perduration particulière comparativement aux séances d'observation précédentes.

8. $17 \%$ ont moins de 18 ans, $30 \%$ plus de 20 ans et seulement $2 \%$ sont âgée's de plus de 25 ans.

9. Dans l'ensemble du texte, le masculin sera employé seul quand il ne nous a jamais été offert l'occasion d'observer une femme accomplir la tâche ou tenir le rôle décrit.

10. Abréviation anglaise de first-person shooter : jeu de tir à la première personne.

11. Osu ! est un jeu sorti en 2007 est disponible gratuitement en téléchargement et développé par Dean « pepy » Herbert. Le but est de respecter le rythme d'un morceau de musique en cliquant sur des cercles en respectant l'ordre numérique selon lequel il apparaissent à l'écran. Plus le/la joueur·euse sera précise et rapide plus les points gagnés seront importants.

12. TrackMania est une série de jeu de course automobile développée par Nadeo dont le premier opus est sorti en 2003 avec de nombreux titres sont disponibles sur consoles de salons et ordinateurs. Si les décors varient selon les versions ce jeu, il reste centré sur la conduite des véhiculent et la recherche de la conduite et de la trajectoire parfaite afin de finir la course le plus rapidement possible. Le mode de jeu le plus visible sur les écrans en LAN partie est celui de la course où le but est de terminer le circuit en tentant de battre le temps d'un véhicule "fantôme " (contrôlé par le logiciel ou en ligne par la personne qui détient le record du meilleur temps pour le mode en ligne). Chaque centimètre de la trajectoire de la voiture menant à des logiques de jeu sur le mode de l'essai/erreur où le perfectionnement est central.

13. Sur l'intérêt d'analyser les photos produites par les organisateurs sur la LAN et ce que ces images disent des conventions qui régissent ces espaces, on pourra se référer à Christine Detrez, La construction sociale du corps, (2002 : 151). 


\section{RÉSUMÉS}

Les LAN sont un univers éminemment masculin où les hommes co-construisent et réaffirment visiblement, ostensiblement même, une figure du joueur viril. Ce travail du genre, individuel et collectif, repose assez largement sur une spécificité masculine supposée du rapport à la technique et à l'habileté de son maniement. Il implique également, ce faisant, de se distinguer, par le verbe notamment, des deux figures repoussoir que sont les femmes et les homosexuels. Entre permanences et résistances, nuances et ajustements aux normes de genre, les femmes doivent, dans cet univers, trouver leur place au sein de la gamme limitée de rôles qui leur sont possibles d'incarner. Si les places varient entre les "filles du staff », les petites amies et les joueuses, toutes ont en commun le care et la gestion des éléments matériels dont elles conservent exclusivement la charge. Elles permettent également, ensemble, d'asseoir une deuxième forme de hiérarchie, interne au groupe des hommes cette fois-ci : entre ceux qui ont pu accéder et peuvent afficher une conjugalité et ceux qui ne le peuvent pas.

LAN parties are an eminently masculine universe where men publically and visibly co-construct and reaffirm the figure of a male virile player. This individual and collective gender display work largely relies on a supposed masculine specificity related to techniques and the skills they require. Performing this male figure also means distinguishing oneself from two counter-figures that are women and homosexuals. In this universe, caught between permanence and resistance, nuances and adjustments to gender norms, women must find their place and, in order to do so, have only a limited number of roles they can - and are allowed to - embody. These roles - both concrete and symbolic - may vary between the "staff girls", the girlfriends and the player-girls, but they all imply a function of "care" and the management of material elements. They also take part in reasserting a hierarchy within men, distinguishing those who succeed in obtaining the favors of women from those who do not.

\section{INDEX}

Mots-clés : genre, jeux vidéo, identité, pratiques culturelles

Keywords : gender, video games, identities, cultural practices

\section{AUTEURS}

\section{JOHANN CHAULET}

CNRS / Université Toulouse Jean-Jaurès, LISST

\section{JESSICA SOLER-BENONIE}

Université Toulouse Jean-Jaurès, LISST 